\title{
Drag Coefficient Parameter Estimation for Aquaculture Systems
}

\author{
Scott C. James · Fearghal O'Donncha
}

Received: 20 July 2018 / Accepted: 5 June 2019

\begin{abstract}
The flow conditions in and around a suspended canopy, resembling those formed by aquaculture structures such as rafts cages and longlines, were modeled using an augmented version of the hydrodynamic model Environmental Fluid Dynamics Code. The model was calibrated using vertical profiles of horizontal velocities, Reynolds stresses, and turbulent kinetic energies obtained from prior laboratory flume experiments. The parameter estimation code, PEST, was used to optimize various model parameters including horizontal momentum diffusivity, vertical eddy viscosity, turbulence closure constants, and, most importantly, depth-dependent drag coefficients. An increasing average drag coefficient was observed with decreasing canopy blockage ratio, and an empirical relationship for the vertical variation of drag coefficient was developed that may be appropriate for use in full-scale models of aquaculture systems. Overall, the calibrated canopy-turbulence parameters and drag-coefficient empiricisms may yield improved predictions of alterations to hydrodynamic and nutrient-transport conditions due to various aquaculture structures. Such predictions will help develop methods to minimize environmental impacts and to increase production from aquaculture farms.
\end{abstract}

Keywords Aquaculture, EFDC, numerical modeling, parameter estimation, turbulence

\section{Introduction}

Aquaculture can have a variety of impacts on the local environment. Aquaculture systems affect the hydrodynamic system by impeding and redirecting flows. The cages, rafts, and lines used in aquaculture systems form suspended canopies that extend downward from the free surface with a gap between the canopy bottom and the sediment

Scott C. James

Baylor University

E-mail: sc_james@baylor.edu

Fearghal O'Donncha

IBM Research - Ireland

E-mail: feardonn@ie.ibm.com 


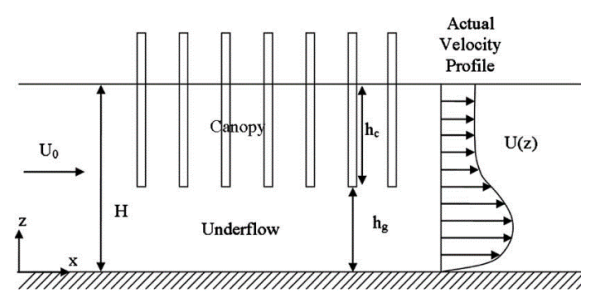

Fig. 1 Schematic of the Plew (2011a) experiments.

bed. Laboratory, field, and numerical studies have shown that suspended canopies impact flow profiles in a number of ways, including reduced velocities within the canopy, accelerated flows beneath the canopy, and the development of shear layer processes at the canopy interface (Zhao et al, 2017; Blanco et al, 1996; Xu and Dong, 2018). Schematically, aquaculture canopies alter the velocity profile as shown in Fig. 1 (Plew et al, 2006; Stevens et al, 2008).

A number of field studies have investigated the hydro-environmental impacts of aquaculture installations. Gibbs et al (1991) observed flow attenuation of up to $70 \%$ in local circulation patterns around long-line mussel dropper farms in Pelorus Sound, New Zealand. The effects of dropper density on flow patterns were investigated by Boyd and Heasman (1998) who observed flow reductions of $86 \%$ and $75 \%$ within droppers spaced 60 and $90 \mathrm{~cm}$, respectively. Pilditch et al (2001) observed a 40\% reduction in flow speed within an $80 \times 50 \mathrm{~m}^{2}$ suspended scallop culture lease in Nova Scotia; Plew et al (2005) demonstrated similar reductions within larger long-line mussel farms and characterized wake development. Newell and Richardson (2014) reported flow attenuations of $75-80 \%$ arising from drag due to culture ropes and supporting infrastructure.

While laboratory investigations of impeded flows historically focused on submerged and emergent canopy configurations (e.g., Naot et al, 1996; Ghisalberti and Nepf, 2004), research on suspended canopies has garnered attention recently. Plew (2011a) conducted flume experiments of suspended canopy configurations and divided velocity and turbulence profiles into three layers: bottom-boundary, canopy-shear, and internalcanopy layers. Similarly, flume studies conducted by Huai and Li (2016) identified maximum velocities at a point between the canopy and bed along with reductions in velocity into and within the canopy; they also divided the vertical flow profile into three layers: vegetation (or canopy) and non-vegetation layers with the latter further subdivided into two layers, each distinctly influenced by the canopy and the bed. Qiao et al (2016) conducted flume experiments of suspended canopy interactions for 18 configurations with varying depth ratios (canopy to water-column depths) and presented a relationship between the depth ratio and the location of the canopy shear layer.

Determining the hydrodynamic properties of these aquaculture structures is important in predicting the transport of nutrients and waste products to and from the system. Simulations can identify ways to improve productivity while minimizing environmental impacts. Panchang et al (1997) developed a two-dimensional numerical model of the distribution of wastes from fish cages in the Gulf of Maine, but did not include the hydrodynamic effects of the aquaculture structures (cage drag). Subsequent efforts included the effects of kelp and scallop cultures on depth-averaged tidal currents and flow patterns in Sungo Bay, China (Grant and Bacher, 2001) and the depth-averaged effects of suspended farms on flows (Plew, 2011b; O'Donncha et al, 
2013). Shi et al (2011) extended the work of Grant and Bacher (2001) in Sungo Bay to three-dimensions and used the amended model to explore the effects of impeded flows on biological processes. Newell and Richardson (2014) combined field measurements and three-dimensional numerical simulations to develop guidelines for minimum ambient flows to provide optimal clearance rates for mussel growth, but did not modify turbulence schemes. A number of modeling studies investigated the effects of canopy installations by incorporating drag terms in both the momentum and turbulence-closure equations. Studies demonstrated sensitivities to bluff-body drag on flows and also vertical transfer of momentum induced by modified turbulent flow processes (O'Donncha et al, 2015b; Zhao et al, 2017). O'Donncha et al (2017) used numerical modeling to investigate the viability of co-locating suspended aquaculture farms with marine hydrokinetic energy devices to exploit the augmented underflow currents generated by the canopy. High-resolution CFD studies of canopy installations have largely been restricted to the flume-scale due to the onerous computational expense at the bay-scale (Tseung et al, 2016).

Field and numerical experiments have demonstrated that aquaculture-induced flows are influenced by the blockage effects of the canopy, which depend both on the canopy density and the lateral spacing between consecutive longitudinal rows of canopy elements. Simulating these processes requires accurate modeling of ambient and amended hydrodynamics. In complex models, this depends on accurate specification of pertinent model parameters (e.g., bottom roughness coefficient, canopy drag, and turbulenceclosure parameters). In the remainder of the paper, considerations towards selecting these parameters are addressed.

\section{Methodology}

\subsection{EFDC Background}

EFDC (Hamrick, 1996, 1992) is a public-domain, open-source, modeling package for simulating three-dimensional (3-D) flow, transport, and biogeochemical processes in surface-water systems. The model is specifically designed to simulate estuaries and subestuarine components (lakes, rivers, tributaries, marshes, wet and dry littoral margins, and coastal regions) and has been applied to a wide range of environmental studies including surface-current processes (O'Donncha et al, 2015a), suspended sediment transport (James et al, 2010), water-quality investigations (James and Boriah, 2010), marine renewable energy (O'Donncha et al, 2017), and canopy flow processes (O'Donncha et al, 2015b).

The equations that form the basis for the EFDC hydrodynamic model (Hamrick, 1992) are based on the continuity and hydrostatic, free-surface Reynolds-averaged Navier-Stokes equations with the Boussinesq approximation, similar to the model of Blumberg and Mellor (2013), except for the solution of the free surface, which is solved with a preconditioned conjugate gradient algorithm. A second-order turbulence-closure model developed by Mellor and Yamada (1982) and modified by Galperin et al (1988) simulates vertical turbulent viscosity in the model. Horizontal diffusion is calculated with the Smagorinsky (1963) formula. EFDC uses a curvilinear-orthogonal grid with a sigma vertical coordinate system. The number of sigma layers is fixed for each model and assigned a constant (often equal) fraction of the flow depth throughout the model domain, where the absolute height of each layer changes with bathymetry and elevation 


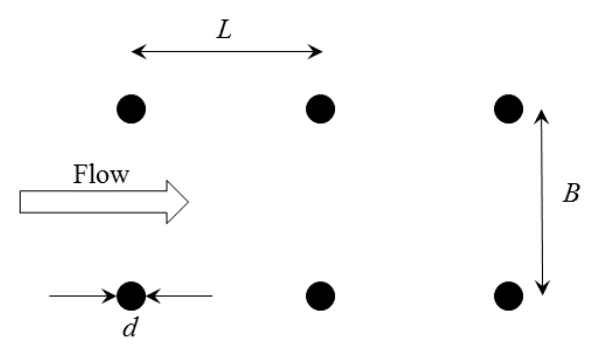

Fig. 2 Schematic showing the parameters defining canopy density.

of the free surface. The code is parallelized using a domain decomposition approach with MPI synchronization between domains (O'Donncha et al, 2014, 2015c), with ongoing research on provisioning through a Cloud offering (O'Donncha et al, 2016a,b).

\subsection{Open-channel Flume Canopy Experiments}

Plew (2011a) conducted two different sets of experiments in laboratory flumes with suspended canopies constructed from arrays of circular cylinders. In each set of experiments, vertical profiles of horizontal velocity, $U$, Reynolds stress, $\left\langle u^{\prime} w^{\prime}\right\rangle$, and turbulent kinetic energy, TKE, were measured. In Flume A, profiles of velocity data were collected with an acoustic Doppler velocimeter (ADV) at 12 locations within the canopy with 12-15 measurements in each profile. In Flume B, two-dimensional (2-D) particle tracking velocimetry (PTV) was used to collect vertical profiles of velocity in $5 \times 5 \mathrm{~mm}^{2}$ grids within the canopy. Seven experiments (A1-A7) were conducted in Flume A and 19 were conducted in the smaller Flume B (B1-B19). Within these two sets of experiments, flow rate, cylinder (dropper) length, and density were varied. All cylinders had diameters $d=9.54 \mathrm{~mm}$. Six of the seven Flume A experiments had nearly identical water depths $(H=250-254 \mathrm{~mm})$ and flow rates $(Q=60-62 \mathrm{~L} / \mathrm{s})$ while 17 of the 19 Flume B experiments had $H=200 \mathrm{~mm}$ and $Q=7-10.5 \mathrm{~L} / \mathrm{s}$. To adjust canopy density in both sets of experiments, cylinder spacing was varied within each row across the channel, $B=50$ or $100 \mathrm{~mm}$, and between rows, $L=100,150$, or $200 \mathrm{~mm}$ (Fig. 2). Cylinders were staggered in Flume A and aligned in Flume B. One Flume A experiment (A2) was excluded from the calibration because it had notably different characteristics (half the flow depth and flow rate). Similarly, four Flume B experiments with the shortest cylinders, B16-B19 (two of which, B18 and B19, also had half the flow depths and flow rates), were excluded from calibration because flow parameters were expected to be different and because nonlinear cylinder-end effects could be dominant. It is also worth noting that the velocities in Flume A were roughly five times greater than those in Flume B $(250-500 \mathrm{~mm} / \mathrm{s}$ versus $50-100 \mathrm{~mm} / \mathrm{s})$. For a full description of the experiments, see Table 1 in the Plew (2011a) paper. 


\subsection{Observations}

In both Flume A and Flume B experiments, vertical profiles of the normalized longitudinal velocity $U$ could be partitioned into three regions: velocities increasing above the bed reaching a maximum at some point between the canopy and the bed; $U$ decreasing into and within the canopy; and velocities approaching a constant value in the upper canopy (q. v. Fig. 4a). An inflexion point was observed near the bottom of the canopy in all velocity profiles. Increased cylinder length, $h_{\mathrm{c}}$, (smaller gap beneath the canopy, $\left.h_{\mathrm{g}}\right)$ resulted in higher velocities within the canopy.

Reynolds shear stresses, $\left\langle u^{\prime} w^{\prime}\right\rangle$, increased linearly from near the bed where they were negative to the bottom of the canopy where they tended toward positive values. Continuing upward into the canopies, $\left\langle u^{\prime} w^{\prime}\right\rangle$ decreased toward zero at the free surface (q. v. Fig. 4b).

Finally, TKE was measured in both flumes; however, there is an important distinction between the TKE measurements in the flumes: those in Flume A were collected with a 3-D ADV while those in Flume B were from 2-D PTV that captured longitudinal and vertical velocities, but not transverse velocity components (i. e., $u$ and $w$, but not $v$ ). (This has an important implication for model calibration discussed later.) These had similar profiles to $U$ except for an additional inflection point near the flume floor due to increased shear from bottom friction (q. v. Fig. 4c). TKE along the profiles decreased upward through the boundary layer before notably increasing at a second inflection point just below the cylinders. This increase was due to the trailing vortices and shear applied to the flow as it was redirected around and under the cylinders. Depending on the experimental parameters, TKE was moderate throughout the canopy at about $1,500-2,500 \mathrm{~mm}^{2} / \mathrm{s}^{2}$ for Flume A and $50-100 \mathrm{~mm}^{2} / \mathrm{s}^{2}$ for Flume B. .

\subsection{Model Development}

EFDC models for flume experiments A1, A3-A7, and B1-B15 were developed. Table 1 lists the flume dimensions and corresponding features implemented in each model. For the larger Flume A, bottom roughness was specified as $0.005 \mathrm{~mm}$ while $0.001 \mathrm{~mm}$ was used for Flume B, values appropriate for PVC and extruded pipe surfaces. Precalibration scoping runs confirmed that these roughness values resulted in bottomlayer velocities consistent with the experimental data. In both models, the time step was $1 / 60^{\text {th }}$ of a second to ensure model stability. In Flume A, canopies were $5.1 \mathrm{~m}$ in length and spanned the full width starting $4.5 \mathrm{~m}$ downstream from the flume entrance. Canopy cells were located in model grid cells ranging from layers 9 through 40 (150 mm-long cylinders in experiment A1) to layers 24 through 40 (50 mm-long cylinders in experiment A7). In Flume B, canopies were $4 \mathrm{~m}$ in length and extended full width starting $1 \mathrm{~m}$ downstream from the flume entrance. In these experiments, canopy cells were located in model grid cells ranging from layers 5 through 40 (175 mm-long cylinders in experiments B1 through B3) to layers 20 through 40 (100 mm-long cylinders in experiments B12 through B15). Model cells containing cylinders were specified with appropriate canopy densities to reflect the longitudinal and transverse spacing specific to each experimental setup as listed in Table 1. In the horizontal plane, model cells were $15 \times 15$ and $10 \times 10 \mathrm{~cm}^{2}$ for Flumes A and B, respectively, while the vertical direction was resolved using 40 equally thick sigma layers for both. Simulated quan- 
Table 1 Flume and EFDC model descriptions.

\begin{tabular}{|c|c|c|}
\hline Feature & Flume A & Flume B \\
\hline Length $(\mathrm{m})$ & 12 & 6 \\
\hline Width $(\mathrm{m})$ & 0.75 & 0.6 \\
\hline$H(\mathrm{~mm})$ & $250-254$ & 200 \\
\hline Roughness $(\mathrm{mm})$ & 0.005 & 0.001 \\
\hline Cell size $\left(\mathrm{cm}^{2}\right)$ & $15 \times 15$ & $10 \times 10$ \\
\hline Horizontal cells & 400 & 360 \\
\hline$\sigma$ layers & 40 & 40 \\
\hline$Q(\mathrm{~L} / \mathrm{s})$ & $60-62$ & $7-10.5$ \\
\hline$h_{\mathrm{c}}(\mathrm{mm})$ & $100,150,200$ & $100,125,150,175$ \\
\hline$L(\mathrm{~mm})$ & $100,150,200$ & $100,150,200$ \\
\hline$B(\mathrm{~mm})$ & 50,100 & 50,100 \\
\hline
\end{tabular}

tities $\left(U,\left\langle u^{\prime} w^{\prime}\right\rangle\right.$, and $\left.T K E\right)$ were extracted at the center of the region where their corresponding measurements were collected; these were used for calibration.

EFDC represents physical obstructions to flow by including an additional drag term in the momentum-conservation equation so that momentum was removed from model cells in which the canopy was specified (i.e., the region of the flume containing cylinders). In this formulation, the model incorporates a momentum sink due to the suspended canopy, $S_{Q}\left(\mathrm{~m} / \mathrm{s}^{2}\right)$, specified according to a bluff-body drag force:

$$
S_{Q}=-\frac{1}{2} C_{\mathrm{D}} a U^{2},
$$

where $C_{\mathrm{D}}(-)$ is the local drag coefficient, $a(1 / \mathrm{m})$ is the projected area of the cylinders per unit volume, and $U(\mathrm{~m} / \mathrm{s})$ is the local flow speed. The effects of canopy elements on turbulence production and dissipation have been widely discussed in the literature, particularly in relation to flows through vegetative canopies (Katul et al, 2004; Poggi et al, 2004). The dominant length scale of turbulence is determined by the dimensions of the obstructing body (the cylindrical canopy element), while turbulence intensity is augmented by the conversion of ambient mean kinetic energy to turbulent kinetic energy (Nepf, 1999). Source/sink terms represent the net changes to turbulent kinetic energy, $S_{k}\left(\mathrm{~m}^{2} / \mathrm{s}^{3}\right)$, and turbulence length scale, $S_{\ell}\left(\mathrm{m}^{3} / \mathrm{s}^{3}\right)$, due to the canopy as:

$$
\begin{gathered}
S_{k}=\frac{1}{2} C_{\mathrm{D}} a\left(\beta_{\mathrm{p}} U^{3}-\beta_{\mathrm{d}} U k\right), \\
S_{\ell}=C_{\ell 4} \ell S_{k},
\end{gathered}
$$

where $k\left(\mathrm{~m}^{2} / \mathrm{s}^{2}\right)$ is the mean-flow kinetic energy and $\beta_{\mathrm{p}}(-)$ is the fraction of $k$ converted to wake-generated kinetic energy by drag, which accounts for the production of wake turbulence and represents the ratio of mean kinetic energy transferred directly into turbulence (not the shear-generated turbulence). $\beta_{\mathrm{d}}(-)$ is the portion of $k$ dissipated through short-circuiting of the turbulence cascade where energy transfers from largescale turbulence to smaller scales. $\ell(\mathrm{m})$ is the turbulence length scale and $C_{\ell 4}(-)$ is the closure constant. Canopy turbulence parameters, $\beta_{\mathrm{p}}, \beta_{\mathrm{d}}$, and $C_{\ell 4}$, must be identified for these aquaculture simulations. For vegetative canopies, Katul et al (2004) suggested $\beta_{\mathrm{p}}=1, \beta_{\mathrm{d}}=1-5$, and $C_{\ell 4}=0.9$, while for wind turbines, Réthoré et al $(2009)$ suggested $\beta_{\mathrm{p}}=0.05, \beta_{\mathrm{d}}=1.5$, and $C_{\ell 4}=1.6$. James et al $(2017)$ suggested $\beta_{\mathrm{p}}=0.96$, $\beta_{\mathrm{d}}=1.38$, and $C_{\ell 4}=3.87$ for hydrokinetic turbines in flume experiments. These values are not directly transferable to aquaculture systems because of density and viscosity 
differences between media, the existence of the free surface, and, of course, the effects of the canopy itself. Instead, these parameters were specified here through calibration to flume data.

\subsection{Calibration Approach}

Flume A and B calibrations were undertaken separately because different flow and turbulence parameters were expected given their distinct experimental conditions. Of the seven Flume A experiments, the six with similar $(H=250-254 \mathrm{~mm})$ water depths were used for calibration (experiment A2 with $H=125 \mathrm{~mm}$ was not considered). Of the 19 Flume B experiments, those with water depth $H=200 \mathrm{~mm}$ and cylinders longer than $h_{\mathrm{c}}=50 \mathrm{~mm}$ were considered, B1-B15. Of those, three were withheld for subsequent validation; B4, B9, and B14, which were selected such that three combinations of $h_{\mathrm{g}}, L$, and $B$ remained in the calibration data set each with an equivalent $h_{\mathrm{g}}$. Two flow parameters $\alpha_{\text {md }}(-)$ and $K_{\mathrm{v}}\left(\mathrm{m}^{2} / \mathrm{s}\right)$ must be specified in EFDC; $\alpha_{\text {md }}$ regulates horizontal momentum diffusion and $K_{\mathrm{v}}$ controls vertical momentum diffusion. Each serves to damp velocity gradients in the respective horizontal or vertical direction (i. e., they govern wake structures). Specifically, $\alpha_{\mathrm{md}}$ is a multiplier on the horizontal stress tensor (Hamrick, 2007, Eqns. (55)-(57)) in the solution of the horizontal momentum conservation equations. It represents subgrid-scale turbulent mixing and also serves to smooth cell-to-cell spatial oscillations in the numerical solution in proportion to the local horizontal strain rate (Smagorinsky, 1963). $K_{\mathrm{v}}$ is the background vertical turbulent eddy viscosity, which appears in the vertical diffusive term in the horizontal momentum and $k-\ell$ turbulence equations (Hamrick, 2007, Eqns. (2), (3), (13), and (14)). These parameters are typically uniquely adjusted for every EFDC model.

For each calibration (all selected experiments for Flumes A or B), global flow ( $\alpha_{\text {md }}$ and $\left.K_{\mathrm{v}}\right)$ and turbulence parameters $\left(\beta_{\mathrm{p}}, \beta_{\mathrm{p}}\right.$, and $\left.C_{\ell 4}\right)$ and experiment-specific dragcoefficient parameters $\left(C_{\mathrm{D}_{n}}\right.$, measured every $5 \mathrm{~mm}$ along cylinders comprising 144 parameters for Flume A and 330 for Flume B) were estimated, requiring every individual flume model to be run for a single calculation of the objective function (i. e., quantification of the differences between measurements and their simulated equivalents). Prior information was supplied in all phases to avoid the tendency for drag coefficients to oscillate along the length of the cylinders when the calibration data did not sufficiently inform the variation of drag coefficients. That is, knowledge that the local drag coefficient should vary smoothly along the cylinder was incorporated into the calibration exercises. All observations (345 in Flume A and 1,423 in Flume B) and prior information (144 in Flume A and 330 in Flume B), which ensured smoother $C_{\mathrm{D}_{n}}$ profiles, were included as calibration data. Because fitting to velocities was considered most important, not only because these data were collected with more precision but because capturing velocity gradients is critical for flow and transport modeling, a weight of 5 was applied to all $U$-profile measurements. Unit weights were applied to $\left\langle u^{\prime} w^{\prime}\right\rangle$ and $T K E$ measurements. Although EFDC's simulated equivalent of the Flume B TKE profiles included the effects of 3-D turbulence while measurements were $2-\mathrm{D}$, it was deemed appropriate to retain these data sets not only for consistency, but to demonstrate that parameter-estimation process provided appropriate fits and parameter values subject to data uncertainty. Moreover, simultaneous calibration to $\left\langle u^{\prime} w^{\prime}\right\rangle$ and inclusion of Flume A resulted in the drag-coefficient empiricisms effectively diluting any potentially deleterious effects from this inconsistency. During scoping runs, overall results 


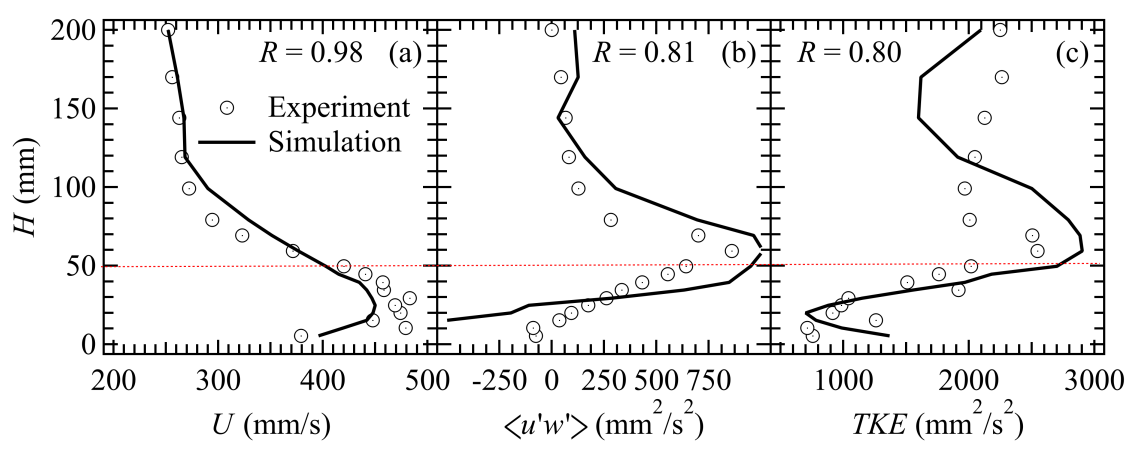

Fig. 3 Comparison of the measured (symbols) and best-fit (a) $U$, (b) $\left\langle u^{\prime} w^{\prime}\right\rangle$, and (c) $T K E$ profiles (curves) for the A1 experiment. The dashed red line indicates the canopy interface.

were not improved when the Flume B TKE data were discarded. Suspicious data points (e.g., step decreases in velocity near the free surface) were de-weighted by a factor of 10. Overall, the simultaneous calibration method required a batch file to sequentially run all 6 (Flume A) or 12 (Flume B) models and save the model outputs as unique files. Calculating the Jacobian (sensitivity matrix) alone required $(155+1) \times 6=936$ EFDC model runs for Flume A and $(335+1) \times 12=4,032$ EFDC model runs for Flume B. Simultaneous calibration ensured common flow and turbulence parameters for each flume while allowing drag coefficients (unique to each experiment) to vary spatially so that they were a function of distance along each cylinder and canopy density ( $B$ and $L$ spacing).

\section{Results \& Discussion}

\subsection{Calibration}

Calibrating the Flume A experiments required 2,052 calls of the batch file $(12,312$ EFDC model runs) while calibrating the Flume B experiments required 3,822 batchfile calls (45,936 EFDC model runs). Example of the fits to vertical profiles of $U,\left\langle u^{\prime} w^{\prime}\right\rangle$, and TKE for experiment A1 are shown in Fig. 3 and for experiment B5 in Fig. 4 along with correlation coefficients, $R$. Fits for the more heavily weighted $U$ profiles were good, while the models tended to over-predict extreme values of $\left\langle u^{\prime} w^{\prime}\right\rangle$ and TKE as expected given the weight emphasis on $U$ data. One reason for such behavior is because the low $K_{\mathrm{v}}$ value appropriate for fitting $U$ profiles increased excursions in the $\left\langle u^{\prime} w^{\prime}\right\rangle$ and TKE profiles. This was deemed acceptable because honoring velocity profiles and gradients is paramount for flow and transport modeling.

It is worth revisiting the implications of the different $T K E$ measurements (3D in Flume A and 2D in Flume B). The ratio of TKEs in Flume A to Flume B is:

$$
\frac{T K E_{3 \mathrm{D}}}{T K E_{2 \mathrm{D}}}=\frac{\frac{1}{2}\left(\left\langle u^{\prime} u^{\prime}\right\rangle+\left\langle v^{\prime} v^{\prime}\right\rangle+\left\langle w^{\prime} w^{\prime}\right\rangle\right)}{\frac{1}{2}\left(\left\langle u^{\prime} u^{\prime}\right\rangle+\left\langle w^{\prime} w^{\prime}\right\rangle\right)} .
$$

The ratios of Flume A to B TKEs computed using (4) at every available measurement location in Flume A are shown in Fig. 5, which illustrates that within the 


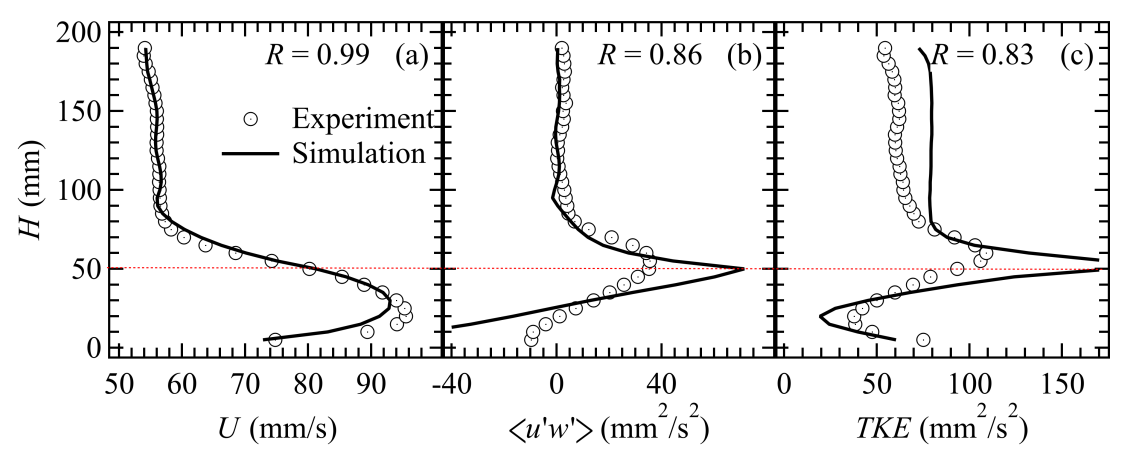

Fig. 4 Comparison of the measured (symbols) and best-fit (a) $U$, b) $\left\langle u^{\prime} w^{\prime}\right\rangle$, and (c) $T K E$ profiles (curves) for the B5 experiment. The dashed red line indicates the canopy interface.

Table 2 Calibrated flow and turbulence parameters.

\begin{tabular}{|c|c|c|}
\hline Parameter & Flume A & Flume B \\
\hline$\alpha_{\mathrm{md}}\left(\mathrm{m}^{2} / \mathrm{s}\right)$ & 1.8 & 1.7 \\
\hline$K_{\mathrm{v}}\left(\mathrm{m}^{2} / \mathrm{s}\right)$ & $7.9 \times 10^{-6}$ & $1.7 \times 10^{-6}$ \\
\hline$\beta_{\mathrm{p}}(-)$ & 1.00 & 0.93 \\
\hline$\beta_{\mathrm{d}}(-)$ & 0.39 & 0.50 \\
\hline$C_{\ell 4}(-)$ & 1.05 & 1.10 \\
\hline
\end{tabular}

canopy, cylinder wakes/vortex streets result in non-isotropic turbulence. In the canopy shear layer and boundary layer, $\left\langle u^{\prime} w^{\prime}\right\rangle$ turbulent structures likely dominate, while $\left\langle u^{\prime} v^{\prime}\right\rangle$ becomes more important in the canopy. Because the cylinder layout was different in Flume B (staggered cylinders in Flume A, in-line in Flume B), the ratios of $T K E /\left(\left\langle u^{\prime} u^{\prime}\right\rangle+\left\langle w^{\prime} w^{\prime}\right\rangle\right)$ were not the same between flumes, ostensibly contributing to the differences between the model and observations. Two conclusions can be drawn: (1) the model tended to over-predict TKE in Flume B (Fig. 4), which is a favorable result because $T K E$ was under-measured (the transverse component was missing) and (2) calibrating the model using partial TKE in Flume B could be responsible for some of the differences in the flow and turbulence parameters between the flumes.

Table 2 lists the calibrated flow and turbulence parameters for each of the flumes. Because of the larger cell size in Flume A, it was not surprising that the calibrated value for $\alpha_{\text {md }}$ was larger as $\alpha_{\text {md }}$ often scales with cell area when implementing an EFDC model. Given the fairly low calibrated values of $K_{\mathrm{v}}$, the vertical eddy viscosities were not vastly different between the two flumes. Calibrated values of the turbulence parameters were similar across flumes. When performing phased calibrations for the Flume B experiments, James et al (2016) obtained flow parameters of $\alpha_{\mathrm{md}}=1.7$ and $K_{\mathrm{v}}=1.5 \times 10^{-6} \mathrm{~m}^{2} / \mathrm{s}$ and turbulence parameters of $\beta_{\mathrm{p}}=0.9, \beta_{\mathrm{d}}=1.4$, and $C_{\ell 4}=3.9$. The values of the turbulence parameters were notably different from those in the present study and may be a result of the less rigorous phased-calibration approach implemented in that earlier study. 


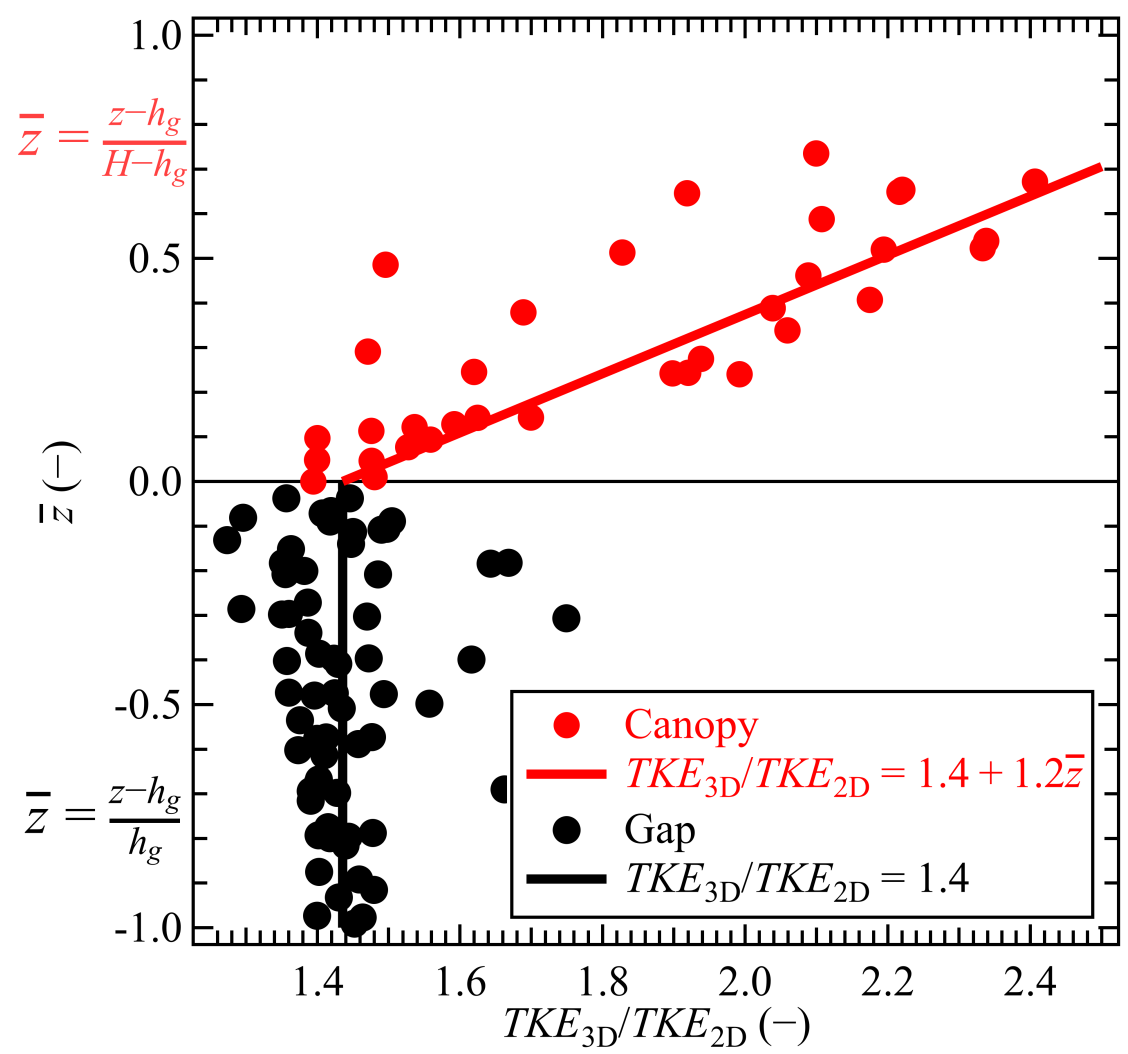

Fig. 5 Ratios of $T K E$ s from Flume A to Flume B. Both $h_{\mathrm{g}}$ and $h_{\mathrm{c}}$ were scaled such that the normalized canopy height ranged from -1 to 0 while the normalized canopy height ranged from 0 to 1 . There was an approximate multiplier of 1.4 beneath the canopy and a linearly increasing relationship into the canopy.

\subsection{Drag Coefficient Relationships}

Trends in the calibrations were analyzed by plotting the averaged depth-dependent drag coefficients (bulk drag coefficients) against the dimensionless canopy density (the product of $a$ and $d$ ). With the results from Flume $\mathrm{A}$ in red and Flume $\mathrm{B}$ in blue, Fig. 6 reveals a trend in both flumes of decreasing bulk drag coefficient with increasing dimensionless canopy density. Fitting a line to all of these data yielded an expression for the bulk drag coefficient as a function of dimensionless aquaculture canopy density:

$$
\bar{C}_{\mathrm{D}}=2.0-67 a d
$$

A similar trend of decreasing bulk drag coefficient was observed in emergent-canopy investigations by Nepf (1999). For submerged and suspended canopies, the finite cylinder has an effect on the drag coefficient (Ghisalberti and Nepf, 2004). On the other hand, in depth-averaged models of emergent canopies, Wu et al (2005) and O'Donncha et al (2013) reported drag coefficients that increased with canopy density. Evidently, the 


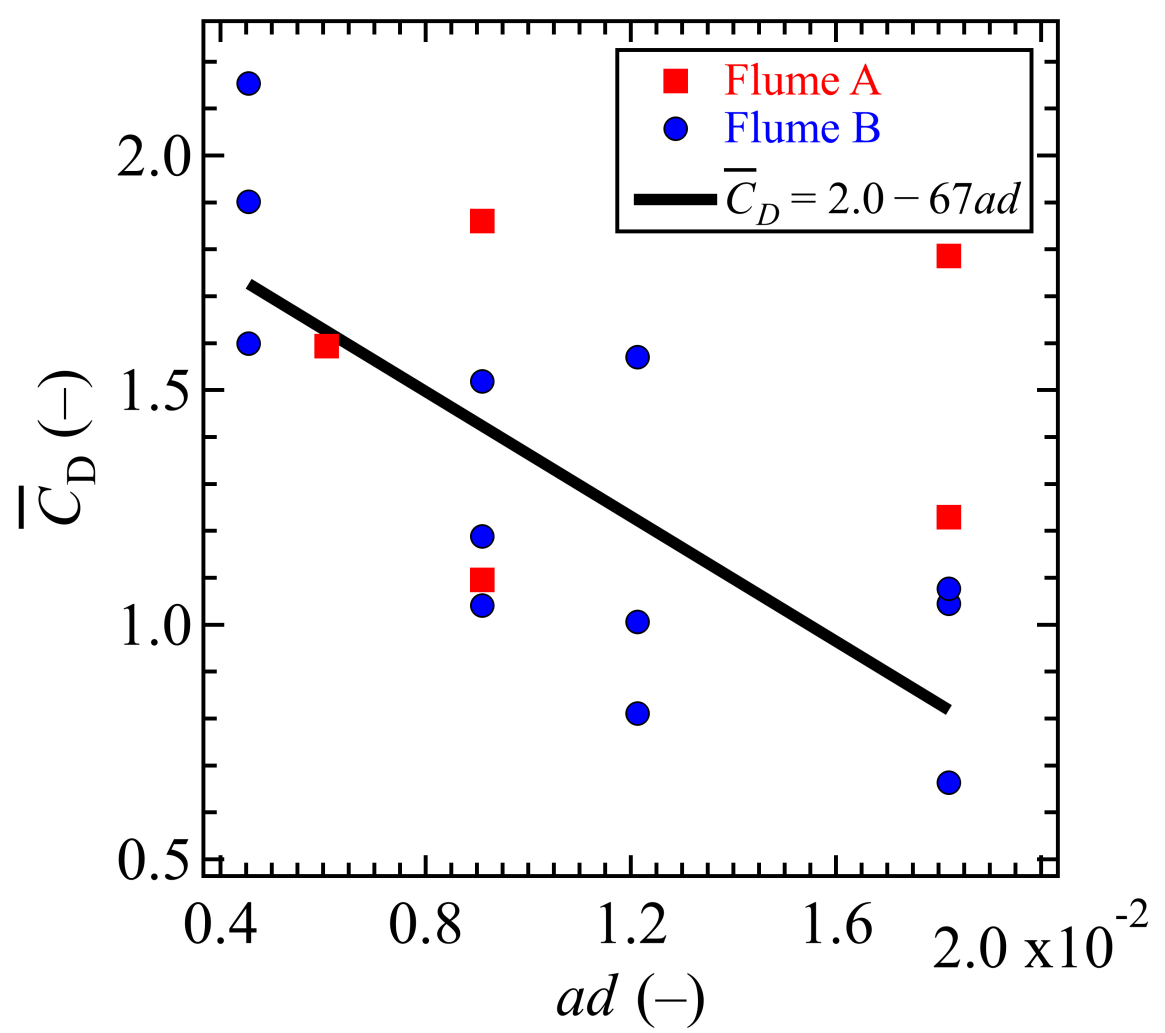

Fig. 6 Bulk drag coefficient, $\bar{C}_{\mathrm{D}}$, as a function of dimensionless canopy density, ad.

specification of drag coefficient to describe canopy behavior is not a trivial matter and requires detailed, bespoke analysis that considers canopy configuration and interaction with flows.

For all 18 experiments used in the calibrations, the depth-varying drag coefficients along normalized lengths of the canopy cylinders, $C_{\mathrm{D}_{n}}$, are plotted in Fig. 7 and revealed a decreasing trend toward the bottom of the cylinders. Averaging across these curves, dividing by $\bar{C}_{\mathrm{D}}$, and fitting a quadratic to the data yielded a function for the drag coefficient along the length of cylinders:

$$
C_{\mathrm{D}}(\zeta)=\bar{C}_{\mathrm{D}}\left(1.2+0.8 \zeta-0.5 \zeta^{2}\right)
$$

where $\zeta(-)$ is the $h_{\mathrm{c}}$-normalized distance from the water surface (this maintains consistency with a bottom-mounted canopy effectively treated as an "inverted" canopy). For comparison to other studies, Fig. 7 includes the drag profiles from Dunn et al (1996) (black curve) and from Ghisalberti and Nepf (2004) (blue curve). Qualitatively, trends are similar with one notable difference: both Dunn et al (1996) and Ghisalberti and Nepf (2004) reported minimum drag coefficients at the top of their canopies (their canopy interface) while in this study the minimum value was at the bottom $(\zeta=0$ or water surface). The key difference, however, is that their canopies were submerged; 


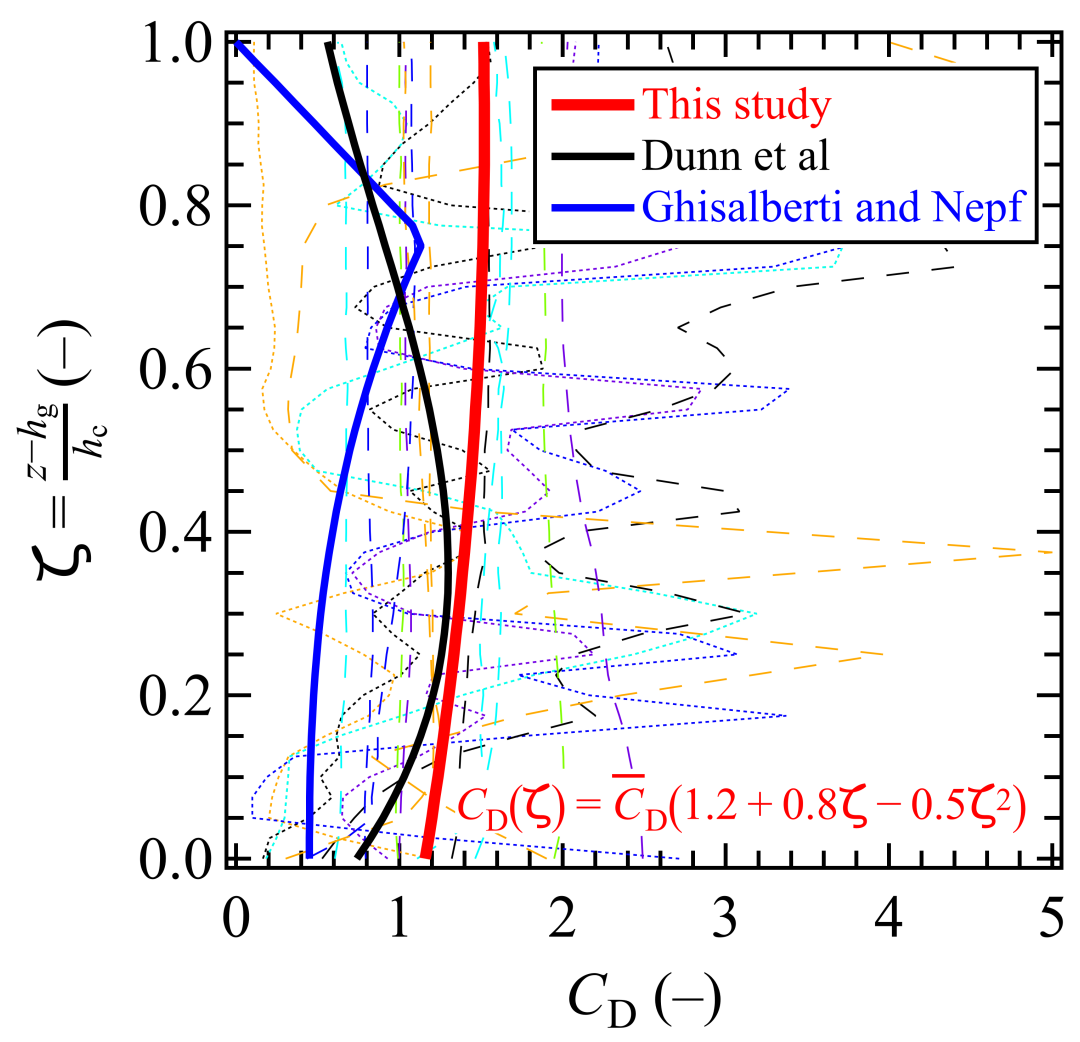

Fig. 7 Depth-dependent drag coefficients normalized along the lengths of the cylinders (dotted curves for Flume A and dashed curves for Flume B). The best fit to the averaged results is indicated with the solid red curve with comparisons to the solutions of Dunn et al (1996) (black curve) and Ghisalberti and Nepf (2004) (blue curve).

they arose from the flume bed, which itself induced additional boundary-layer drag, compared to this suspended canopy configuration with a flowing water surface.

Together, relationships (5) and (6) specify drag coefficients that can vary both spatially across model cells and vertically across model layers depending on the canopy density and the vertical position within the canopy, respectively. These serve as a starting point for scaling the model results to other aquaculture systems, particularly those having similar Reynolds numbers below the canopy $\left(1.8 \times 10^{3}<R e<5.7 \times 10^{4}\right)$.

\subsection{Sensitivities}

PEST calculates sensitivities as the change in the objective function per unit change in a parameter; however, these are complicated by the weighting scheme and magnitudes (if not normalized) of the measurements while also being only locally applicable in parameter space (e.g., as a parameter goes to zero, its sensitivity also goes to zero; as $C_{\mathrm{D}} \rightarrow 0$, the canopy "disappears"). Nevertheless, a few observations are notewor- 
thy regarding Table 3, which lists the sensitivities of the simulated $U,\left\langle u^{\prime} w^{\prime}\right\rangle$, and $T K E$ profiles to the flow, turbulence, and drag parameters. Note that drag-coefficient sensitivities are presented as averages (144 $C_{\mathrm{D}_{n}}$ components for Flume A and 330 for Flume B). It was not surprising that in both calibrations, simulated vertical profiles were more sensitive to background vertical eddy viscosity, $K_{\mathrm{v}}$, than to the horizontal momentum diffusion coefficient, $\alpha_{\text {md }}$. It was also unsurprising that simulated TKE profiles were more sensitive to the turbulence parameters $\left(\beta_{\mathrm{p}}, \beta_{\mathrm{d}}\right.$, and $\left.C_{\ell 4}\right)$ than were $U$ or $\left\langle u^{\prime} w^{\prime}\right\rangle$ profiles. Interestingly, the turbulence-closure constant, $C_{\ell 4}$, controlling the turbulence length scale was the parameter to which simulated vertical profiles were most sensitive because as it increased, momentum transfer between layers increased as TKE dissipated (Nepf and Vivoni, 2000). The upshot of this analysis is that if a model is not simulating profiles with sufficient accuracy, the first parameter that should be adjusted is $C_{\ell 4}$, followed by $K_{\mathrm{v}}$ and the other turbulence parameters. Finally, the goodness of fit was quantified through correlation coefficients, $R$, for the profiles of each quantity against measured data. Results demonstrate highest correlation for the $U$ profiles because of the preferential weights given those data during calibration.

Table 3 Flume A/B parameter sensitivities and average correlation coefficients, $R$.

\begin{tabular}{|c|c|c|c|}
\hline Parameter & $U$ & $\left\langle u^{\prime} w^{\prime}\right\rangle$ & $T K E$ \\
\hline$\alpha_{\mathrm{md}}$ & $0.95 / 0.67$ & $1.38 / 0.32$ & $1.67 / 0.32$ \\
\hline$K_{\mathrm{v}}$ & $6.93 / 4.14$ & $7.06 / 3.74$ & $7.26 / 2.21$ \\
\hline$\beta_{\mathrm{p}}$ & $4.21 / 0.90$ & $1.72 / 2.69$ & $4.23 / 11.26$ \\
\hline$\beta_{\mathrm{d}}$ & $3.5 / 1.06$ & $5.08 / 1.22$ & $4.28 / 27.15$ \\
\hline$C_{\ell 4}$ & $15.60 / 23.34$ & $16.99 / 71.24$ & $80.40 / 266.08$ \\
\hline$\tilde{C}_{\mathrm{D}}$ & $2.47 / 3.90$ & $1.36 / 2.53$ & $1.68 / 1.98$ \\
\hline \hline$R$ & $0.98 / 0.95$ & $0.91 / 0.79$ & $0.81 / 0.74$ \\
\hline
\end{tabular}

\subsection{Validation}

Experiments B4, B9, and B14 were used to validate the estimated parameters and the $C_{\mathrm{D}}$ empiricisms. Each experiment was simulated using the calibrated flow and turbulence parameters in addition to specifying the drag coefficients according to (5) and (6). Correlation coefficients, $R$, were quite high for the $U$ profiles with sequentially poorer model reproductions of $\left\langle u^{\prime} w^{\prime}\right\rangle$ and TKE. With the increased weights applied to $U$-profile data, the model simulated sharper gradients in the $\left\langle u^{\prime} w^{\prime}\right\rangle$ and $T K E$ profiles, which could be smoothed by increasing $K_{\mathrm{v}}$, but this would come at the expense of a poorer fit to the $U$ profile. Given that it is most important for a flow and transport model to honor velocity gradients, this validation was acceptable. Fig. 8 shows how these simulations matched the measured profiles previously withheld during calibration. Results are clearly not as good as those from calibration, but the general trends are honored.

\section{Conclusions}

In two different laboratory flumes, Plew (2011a) conducted sets of experiments to quantify the effects of aquaculture canopy length and density on the local flow field 


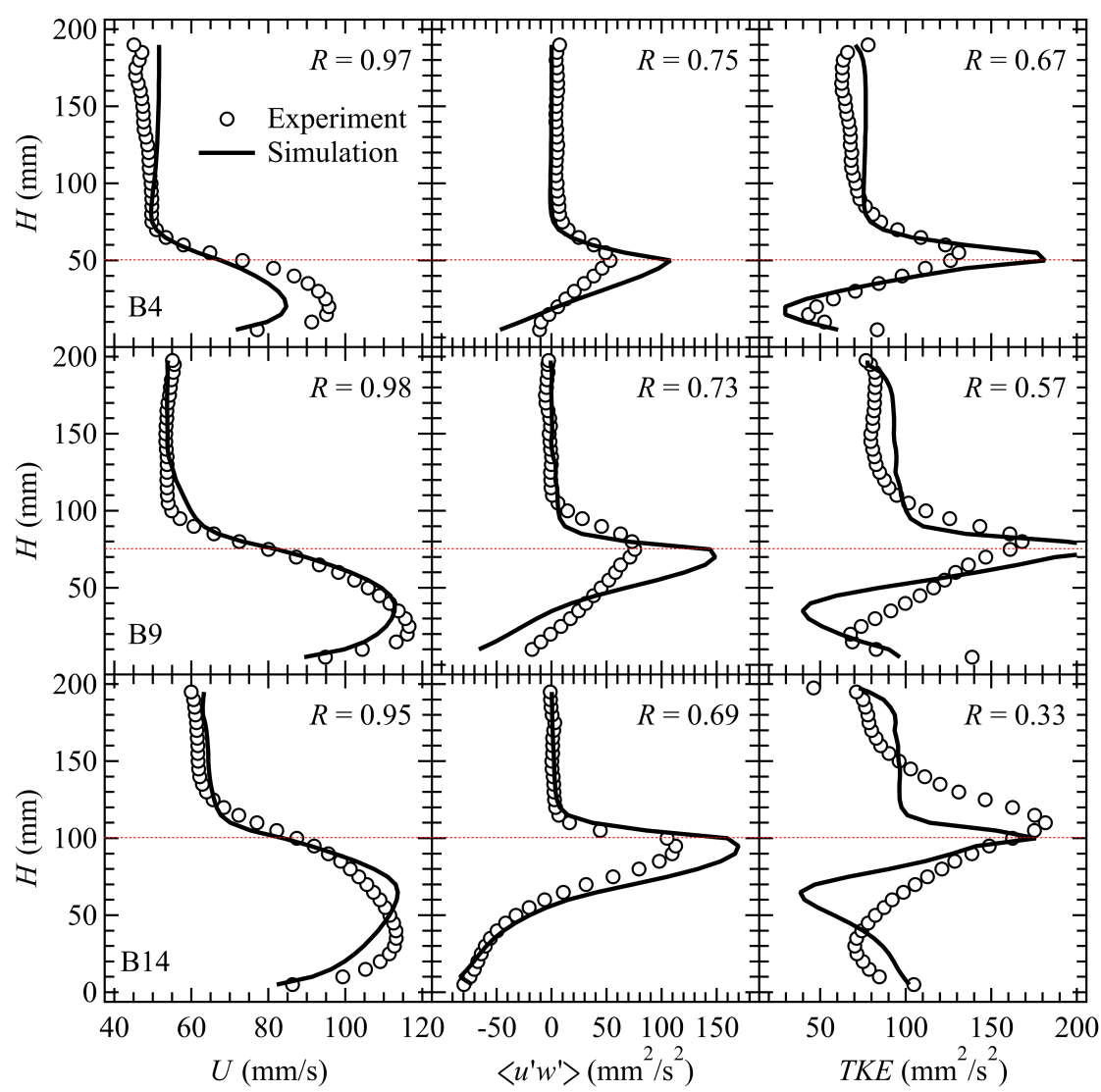

Fig. 8 Validation: Comparison of the measured (symbols) and simulated (curves) $U$ (left column), $\left\langle u^{\prime} w^{\prime}\right\rangle$ (middle column), and TKE (right column) profiles when the calibrated parameters for Flume $\mathrm{B}$ and $C_{\mathrm{D}}$ empiricisms (5) and (6) were supplied to models of experiments B4 (top row), B9 (middle row), and B14 (bottom row). The dashed red lines indicate the canopy interfaces.

by measuring velocity components as well as their fluctuations. EFDC models of these flume experiments were built and supplied to the parameter-estimation code, PEST. Simultaneous calibrations to the two sets of flume experiments were undertaken to estimate flow and turbulence parameters specific to each flume along with universal depth-dependent drag coefficients determined across both flumes (six experiments in Flume A and 12 in Flume B) by matching simulated and measured vertical profiles of horizontal velocities, Reynolds stresses, and turbulent kinetic energies. Trends of decreasing average drag coefficient with increasing canopy blockage ratio (canopy density defined by longitudinal and lateral spacing) and decreasing drag coefficient along the length of the cylinders were observed. Importantly, the turbulence-closure constant, $C_{\ell 4}$ was the parameter to which simulated vertical profiles were most sensitive (as it increased, momentum transfer between layers increased as TKE dissipated. Because cylinder lengths and densities were varied among experiments in each flume, the 
calibrated depth-dependent drag coefficients were described with an empirical relationship that was a function of both distance along the cylinder and cylinder density. This empirical relationship for the canopy-density-dependent vertical variation of drag coefficient as a function of canopy density could be used in full-scale models of aquaculture systems. The calibrated canopy-turbulence parameters may yield improved predictions of the hydrodynamic and material transport conditions resulting from the aquaculture structure. In turn, these predictions will help develop methods to minimize environmental impacts and increase productivity of aquaculture farms.

\section{Acknowledgements}

Elements of this research received funding from the European Union's Horizon 2020 research and innovation program under grant agreement No.773330. The manuscript was vastly improved because of thorough critiques offered by two anonymous reviewers.

\section{References}

Blanco J, Zapata M, Moroño Á (1996) Some aspects of the water flow through mussel rafts. Scientia Marina 60(2-3):275-282

Blumberg AF, Mellor GL (2013) A Description of a Three-Dimensional Coastal Ocean Circulation Model, American Geophysical Union, pp 1-16. DOI 10.1029/CO004p0001

Boyd AJ, Heasman KG (1998) Shellfish mariculture in the Benguela System: Water flow patterns within a mussel farm in Saldanha Bay, South Africa. Journal of Shellfish Research 17(1):25-32

Dunn C, Lopez F, Garcia M (1996) Mean flow and turbulence in a laboratory channel with simulated vegetation. Tech. rep., University of Illinois at Urbana-Champaign, Department of Civil Engineering

Galperin B, Kantha LH, Hassid S, Rosati A (1988) A quasi-equilibrium turbulent energy model for geophysical flows. Journal of the Atmospheric Sciences 45(1):5562, DOI 10.1175/1520-0469(1988)045<0055:AQETEM $>2.0$.CO;2

Ghisalberti M, Nepf HM (2004) The limited growth of vegetated shear layers. Water Resourources Research 40(7):1-12

Gibbs MM, James MR, Pickmere SE, Woods PH, Shakespeare BS, Hickman HW, Illingworth J (1991) Hydrodynamic and water column properties at six stations associated with mussel farming in Pelorus Sound. New Zealand Journal of Marine and Freshwater Research 25(3):239-254

Grant J, Bacher C (2001) A numerical model of flow modification induced by suspended aquaculture in a Chinese bay. Canadian Journal of Fisheries and Aquatic Sciences 58:1003-1011

Hamrick J (1992) A three-dimensional environmental fluid dynamics computer code: Theoretical and computational aspects. Tech. rep., Virginia Institute of Marine Science

Hamrick J (2007) The Environmental Fluid Dynamics Code: Theory and computation. Tech. rep., US EPA, Fairfax, VA

Hamrick JM (1996) User's manual for the environmental fluid dynamics computer code. Tech. rep., Virginia Institute of Marine Science 
Huai W, Li C (2016) Longitudinal dispersion in open channel flow with suspended canopies. Water Science and Technology 74(3):722-728

James SC, Boriah V (2010) Modeling algae growth in an open-channel raceway. Journal of Computational Biology 17(7):895-906

James SC, Jones CA, Grace MD, Roberts JD (2010) Advances in sediment transport modelling. Journal of Hydraulic Research 48(6):754-763

James SC, O'Donncha F, Plew DR (2016) Calibration of a 3D hydrodynamic aquaculture model. In: OCEANS'16, Monterey, California, pp 1-7

James SC, Johnson EL, Barco J, Roberts JD (2017) Simulating current-energy converters: SNL-EFDC model development, verification, and parameter estimation. Renewable Energy DOI 10.1016/j.renene.2017.07.020

Katul GG, Mahrt L, Poggi D, Sanz C (2004) One- and two-equation models for canopy turbulence. Boundary-Layer Meteorology 113:81-109

Mellor GL, Yamada T (1982) Development of a turbulence closure model for geophysical fluid problems. Review of Geophysics 20(4):851-875

Naot D, Nezu I, Nakagawa H (1996) Hydrodynamic behaviour of partly vegetated open channels. Journal of Hydraulic Engineering 122(11):625-633

Nepf HM (1999) Drag, turbulence and diffusion in flow through emergent vegetation. Water Resources Research 35(2):479-489

Nepf HM, Vivoni ER (2000) Flow structure in depth-limited, vegetated flow. Journal of Geophysical Research 105(C12):28,547-28,557

Newell CR, Richardson J (2014) The effects of ambient and aquaculture structure hydrodynamics on the food supply and demand of mussel rafts. Journal of Shellfish Research 33(1):257-272

O'Donncha F, Hartnett M, Nash S (2013) Physical and numerical investigation of the hydrodynamic implications of aquaculture farms. Aquacultural Engineering 52:1426, DOI 10.1016/j.aquaeng.2012.07.006

O'Donncha F, Ragnoli E, Suits F (2014) Parallelisation study of a three-dimensional environmental flow model. Computers \& Geosciences 64:96-103

O’Donncha F, Hartnett M, Nash S, Ren L, Ragnoli E (2015a) Characterizing observed circulation patterns within a bay using HF radar and numerical model simulations. Journal of Marine Systems 142:96-110

O'Donncha F, Hartnett M, Plew DR (2015b) Parameterizing suspended canopy effects in a three-dimensional hydrodynamic model. Journal of Hydraulic Research 53(6):714-727, DOI 10.1080/00221686.2015.1093036

O'Donncha F, James SC, O'Brien N, Ragnoli E (2015c) Parallelisation of hydroenvironmental model for simulating marine current devices. In: OCEANS'15, Washington D.C., pp 1-7

O'Donncha F, Ragnoli E, Venugopal S, James SC, Katrinis K (2016a) On the efficiency of executing hydro-environmental models on Cloud. Procedia Engineering 154:199206, DOI 10.1016/j.proeng.2016.07.447

O'Donncha F, Venugopal S, James SC, Ragnoli E (2016b) Deploying and optimizing performance of a 3D hydrodynamic model on Cloud. In: OCEANS'16, Monterey, California, pp 1-7

O'Donncha F, James SC, Ragnoli E (2017) Modelling study of the effects of suspended aquaculture installations on tidal stream generation in Cobscook Bay. Renewable Energy 102:65-76

Panchang V, Cheng G, Newell C (1997) Modeling hydrodynamics and aquaculture waste transport in coastal Maine. Estuaries 20(1):14-41 
Pilditch CA, Grant J, Bryan KR (2001) Seston supply to sea scallops (Placopecten magellanicus) in suspended culture. Canadian Journal of Fisheries and Aquatic Sciences $58(2): 241-253$

Plew DR (2011a) Depth-averaged drag coefficient for modeling flow through suspended canopies. Journal of Hydraulic Engineering 137(2):234-247, DOI 10.1061/(ASCE)HY.1943-7900.0000300

Plew DR (2011b) Shellfish farm-induced changes to tidal circulation in an embayment, and implications for seston depletion. Aquaculture Environment Interactions 1:201214

Plew DR, Stevens CL, Spigel RH, Hartstein ND (2005) Hydrodynamic implications of large offshore mussel farms. IEEE Journal of Oceanic Engineering 30(1):95-108

Plew DR, Spigel RH, Stevens CL, Nokes RI, Davidson MJ (2006) Stratified flow interactions with a suspended canopy. Environmental Fluid Mechanics 6(6):519-539

Poggi D, Porporato A, Ridolfi L, Albertson J, Katul G (2004) The effect of vegetation density on canopy sub-layer turbulence. Boundary-Layer Meteorology 111(3):565587

Qiao J, Delavan S, Nokes R, Plew D (2016) Flow structure and turbulence characteristics downstream of a spanwise suspended linear array. Environmental Fluid Mechanics 16(5):1021-1041

Réthoré PEM, Sørensen NN, Bechmann A, Zahle F (2009) Study of the atmospheric wake turbulence of a CFD actuator disc model. In: 2009 European Wind Energy Conference and Exhibition

Shi J, Wei H, Zhao L, Yuan Y, Fang J, Zhang J (2011) A physical-biological coupled aquaculture model for a suspended aquaculture area of china. Aquaculture 318(34):412-424

Smagorinsky J (1963) General circulation experiments with the primitive equations, Part I: The basic experiment. Monthly Weather Review 91(3):99-164

Stevens C, Plew DR, Hartstein N, Fredriksson D (2008) The physics of open-water shellfish aquaculture. Aquacultural Engineering 38(3):145-160

Tseung HL, Kikkert GA, Plew D (2016) Hydrodynamics of suspended canopies with limited length and width. Environmental Fluid Mechanics 16(1):145-166

Wu W, Shields Jr FD, Bennett SJ, Wang SSY (2005) A depth-averaged twodimensional model for flow, sediment transport, and bed topography in curved channels with riparian vegetation. Water Resources Research 41(W03015):1-15

Xu TJ, Dong GH (2018) Numerical simulation of the hydrodynamic behaviour of mussel farm in currents. Ships and Offshore Structures pp 1-12

Zhao F, Huai W, Li D (2017) Numerical modeling of open channel flow with suspended canopy. Advances in Water Resources 105:132-143, DOI 10.1016/j.advwatres.2017.05.001 